\title{
PRICING THE ZERO-COUPON BOND AND ITS FAIR PREMIUM UNDER A STRUCTURAL CREDIT RISK MODEL WITH JUMPS
}

\author{
YINGHUI DONG, * Suzhou University and Suzhou University of Science and Technology \\ GUOJING WANG, ${ }^{* * *}$ Suzhou University \\ RONG WU, ${ }^{* * *}$ Nankai University
}

\begin{abstract}
In this paper we consider a structural form credit risk model with jumps. We investigate the credit spread, the price, and the fair premium of the zero-coupon bond for the proposed model. The price and the fair premium of the bond are associated with the Laplace transform of default time and the firm's expected present market value at default. We give sufficient conditions under which the Laplace transform and the expected present market value of a firm at default are twice continuously differentiable. We derive closedform expressions for them when the jumps have a hyperexponential distribution. Using the closed-form expressions, we obtain numerical solutions for the default probability, the credit spread, and the fair premium of the bond.
\end{abstract}

Keywords: Credit spread; default probability; hyperexponential distribution; fair premium rate; structural credit risk model; zero-coupon bond

2010 Mathematics Subject Classification: Primary 60J75; 44A10

\section{Introduction}

Defaultable zero-coupon bond and credit default swap (CDS) are important credit derivatives. The fair premium of the bond is associated with its CDS. It is of special interest to price these derivatives in credit risk theory. The structural form model, initially proposed in Black and Scholes (1973), Merton (1974), and Black and Cox (1976), could give an intuitive understanding for the credit risk by specifying a firm's value process. A commonly used method for investigating the credit risk in structural form models is the first passage time approach, which specifies the default as the first time that the firm's value falls below a threshold level. See, for example, Black and Cox (1976), Longstaff and Schwartz (1995), Collin-Dufresne and Goldstein (2001).

In some earlier structural form models, the firm's market value process is assumed to follow a diffusion process. This assumption leads to zero limiting credit spreads, which contradicts the empirical observation that credit spreads do not vanish as maturity decreases to 0 . To overcome this shortcoming, many authors consider structural form credit risk models in which the firm's market value process contains jumps. See, for example, Zhou (2001), Hilberink and Rogers (2002), and Le Courtois and Quittard-Pinon (2006). Following the idea of these papers, we also consider a structural form model with jumps. Using the first passage time approach, we

Received 14 October 2010; revision received 19 February 2011.

* Postal address: Department of Mathematics and Center for Financial Engineering, Suzhou University, Suzhou 215006, P. R. China.

** Email address: dongyinghui1030@163.com

*** Postal address: Department of Mathematics and LPMC, Nankai University, Tianjin 300071, P. R. China. 
investigate the credit spread, the price, and the fair premium of the bond under the proposed model.

All random variables considered in this paper are defined on the filtered complete probability space $\left\{\Omega, \Im,\left\{\Im_{t}\right\}, P\right\}$. We assume that the total market value of a firm under the pricing probability measure $\mathrm{P}$ is given by

$$
V(t)=V_{0} \exp \left[\mu t+\sigma W(t)+\sum_{i=0}^{N(t)} Z_{i}\right]
$$

for $t \geq 0$, where $\mu, \sigma$, and $V_{0}$ are positive constants, and $V_{0}$ represents the initial market value of the firm; $\{W(t)\}$ is a standard Brownian motion; $\{N(t)\}$ is a Poisson process with parameter $\lambda>0$; and the random variables $\left\{Z_{i}\right\}$ are independent and identically distributed with common distribution $F$. It is assumed that $\{W(t)\},\{N(t)\}$, and $\left\{Z_{i}\right\}$ are independent. This means that $\sum_{i=0}^{N(t)} Z_{i}$ is a compound Poisson process with intensity $\lambda>0$ and jump size distribution $F$.

For simplicity, we let the constant $K>0$ denote the debt of the firm. Set $u=-\ln \left(K / V_{0}\right)$. Following Longstaff and Schwartz (1995) we define the default time as

$$
\tau_{u}=\inf \{t: V(t) \leq K\}
$$

with $\tau_{u}=\infty$ if $V(t)>K$ for all $t \geq 0$. We call the constant $K$ the default threshold level. As in Black and Cox (1976), we let the default threshold level $K$ be an exponential function of time $t$. However, this choice does not bring any essential extension of mathematics in the following discussions.

Consider the jump diffusion process defined by

$$
X(t)=u+\mu t+\sigma W(t)+\sum_{i=0}^{N(t)} Z_{i}, \quad t \geq 0 .
$$

Then, we have

$$
\tau_{u}=\inf \{t: X(t) \leq 0\}
$$

with $\tau_{u}=\infty$ if $X(t)>0$ for all $t \geq 0$. Given $T>0$, the default probability of the firm in $[0, T]$ is defined as $\Psi(u, T)=\mathrm{P}\left(\tau_{u} \leq T\right)$.

If $F(0)=1$, the process $X(t)$ given in (1.2) reduces to the classical risk process perturbed by diffusion introduced in Gerber (1970) in insurance mathematics. In this case, $u \geq 0$ is the initial surplus of the insurer and the default time $\tau_{u}$ above is, in fact, the time of ruin; see Dufresne and Gerber (1991). Ruin theory for Gerber's classical risk process perturbed by diffusion has been extensively studied in risk theory. See, for example, Dufresne and Gerber (1991), Furrer and Schmidli (1994), Gerber and Shiu (1998), Wang and Wu (2000), Tsai and Willmot (2002), and Chiu and Yin (2003).

Using similar processes to models (1.1) and (1.2), Chen and Panjer (2009) investigated connections between ruin theory and credit risk for a structural form credit risk process with jumps, so that the results developed in ruin theory can be used to develop analogous results in credit risk. Zhou (2001) considered a structural credit risk model with jumps, and provided a simple Monte Carlo algorithm to evaluate bond prices and credit spread when the jumps have normal distribution. Kou and Wang (2003) and Le Courtois and Quittard-Pinon (2006) derived closed-form solutions for the Laplace transform of the first passage times that is associated with the jump diffusion process (1.2) when the jumps are double-exponentially distributed by using 
the memoryless property of the exponential distribution. Ramezani and Zeng (2007) considered an asset process similar to process (1.1). Using a maximum likelihood estimation procedure, they found that the asset process for both individual stocks and the indexes of the S\&P-500 and the NASDAQ with jumps having double-exponential distribution performs better than the case with normally distributed jumps or without jumps. There are a number of works that study the double-exponential jump diffusion model; see Kou (2002), Kou and Wang (2003), (2004), Huang and Huang (2003), and Huang and Zhou (2008), to name only a few. Intuitively, jumps of the firm's value process are triggered if unexpected information or events are revealed, and there might be a variety of information. Motivated by the work of Lindskog and McNeil (2003), we assume that different varieties of unexpected information or events arrive as independent Poisson processes, and that the jump sizes caused by the same type of information are independent and have a common distribution. Hence, the jump components of the firm's value process caused by unexpected information or events can be seen to be a sum of some independent compound Poisson-distributed random variables, and, therefore, itself compound Poisson distributed. If all types of jump size follow double-exponential distributions, then the firm's value process is a hyperexponential jump diffusion model, which is a generalization of double-exponential jump diffusion model. In fact, the credit risk process (1.1) with jumps having a hyperexponential distribution may be more flexible, and it can well approximate many heavy-tailed distributions, including exponential-tail and power-tail distributions. Hence, it can be used to incorporate the uncertainty of the heaviness of the asset return tails.

The aim of this paper is to provide a method to value the price and the fair premium of the zerocoupon bond under credit risk process (1.1). Specifically, we present closed-form expressions for them when the jumps have a hyperexponential distribution. The rest of this paper is organized as follows. In Section 2 we present the connections between the price and the fair premium of the zero-coupon bond, the Laplace transform of default time, and the firm's expected present value at default. In Section 3 we give sufficient conditions under which the Laplace transform and the firm's expected present value at default are twice continuously differentiable and derive closed-form expressions for them when the jumps have a hyperexponential distribution. Using the closed-form expressions, we obtain numerical solutions for the credit spread, the default probability, and the fair premium of the bond in Section 4 . Section 5 provides some conclusions.

\section{The price and the fair premium of the zero-coupon bond}

Let $\delta>0$. The Laplace transform of $\tau_{u}$ is defined as $L(u)=\mathrm{E}\left[\mathrm{e}^{-\delta \tau_{u}}\right]$. We have

$$
L(u)=1 \text { for } u \leq 0 .
$$

The density of the default time $\tau_{u}$ can be obtained by inverting its Laplace transform $L(u)$.

Let $\phi(u)=\mathrm{E}\left[V\left(\tau_{u}\right) \mathrm{e}^{-\delta \tau_{u}} \mathbf{1}\left(\tau_{u}<\infty\right)\right]$ denote the expected presented market value of a firm at default time, where $\delta>0$ is the discounted factor. It is easy to see that

$$
\phi(u)=V_{0} \quad \text { for } u \leq 0 .
$$

Some exact expressions for $\phi(u)$ are obtained when the jumps in the credit risk process (1.1) have a double-exponential distribution or when the jump diffusion process $X(t)$ of (1.2) is replaced by a Lévy process with no upward jumps, a so-called spectrally negative Lévy process. See Le Courtois and Quittard-Pinon (2006) and Hilberink and Rogers (2002).

The price and the fair premium of a defaultable zero-coupon bond are associated with the functions $L(u)$ and $\phi(u)$. Given a short constant rate of interest $r>0$, the fair price $B(0, T)$ 
of a defaultable zero-coupon bond at time 0 with maturity $T$ and unit face value is given by

$$
B(0, T)=\mathrm{e}^{-r T} \mathrm{P}\left(\tau_{u}>T\right)+\frac{R \mathrm{E}\left[\mathrm{e}^{-r \tau_{u}} V\left(\tau_{u}\right) \mathbf{1}\left(\tau_{u} \leq T\right)\right]}{K},
$$

where $R \in[0,1]$ is a constant. The first term on the right-hand side of $(2.3)$ is the present value of the bond if there is no default occurring before time $T$. The second term is the expected present value of what the bondholders receive upon default (if this happens before maturity). The bondholder receives the fraction $R V\left(\tau_{u}\right) / K$ of the face value in case of a default. We can interpret $1-R$ as the fraction of the liquidity cost of the firm when default occurs.

The credit spread corresponding to $B(0, T)$ is denoted as $\eta_{T}$; it is the solution to the equation $B(0, T)=\mathrm{e}^{-\left(r+\eta_{T}\right) T}$. Hence, we have

$$
\eta_{T}=-\frac{\ln (B(0, T))}{T}-r
$$

Given $B(0, T)$, we can obtain $\eta_{T}$ from (2.4).

In a credit default swap (CDS) contract written on the firm's zero-coupon bond, we assume that the insurance seller will continuously receive the premium at rate $c$ until the firm goes bankrupt and will cover the loss of the insurance buyer in the event of credit default. This means that the price $\operatorname{CDS}(0, T)$ of the contract with notional value 1 , continuous premium rate payments $c$, and maturity $T$ is

$$
\operatorname{CDS}(0, T)=\mathrm{E}\left[\left(1-\frac{R V\left(\tau_{u}\right)}{K}\right) \mathrm{e}^{-r \tau_{u}} \mathbf{1}\left(\tau_{u} \leq T\right)\right]-\mathrm{E}\left[\int_{0}^{T} c \mathrm{e}^{-r t} \mathbf{1}\left(\tau_{u}>t\right) \mathrm{d} t\right] .
$$

The first term on the right-hand side of (2.5) is the expected present value of the loss of the bond. The second term is the expected present value of the premium paid by the bondholder till default occurs. The fair premium rate $C_{T}$ should make the value of $\operatorname{CDS}(0, T) 0$. Hence, from (2.5) we obtain

$$
C_{T}=\frac{\mathrm{E}\left[\left(1-R V\left(\tau_{u}\right) / K\right) \mathrm{e}^{-r \tau_{u}} \mathbf{1}(\tau \leq T)\right]}{\mathrm{E}\left[\int_{0}^{T} \mathrm{e}^{-r t} \mathbf{1}\left(\tau_{u}>t\right) \mathrm{d} t\right]}=: \frac{A_{1}(u, T)}{A_{2}(u, T)} .
$$

Since there are no exact results for the price of the bond and for the fair premium under credit risk process (1.1), following Hilberink and Rogers (2002) and Le Courtois and Quittard-Pinon (2006), we consider the Laplace transforms of the price $B(0, T)$ and the Laplace transforms of $A_{1}(u, T)$ and $A_{2}(u, T)$. By inverting these Laplace transforms we could obtain the price of the bond and the fair premium.

The Laplace transform of $B(0, T)$ is given by

$$
\begin{aligned}
\hat{B}(\gamma) & =\int_{0}^{\infty} \mathrm{e}^{-\gamma T} B(0, T) \mathrm{d} T \\
& =\int_{0}^{\infty} \mathrm{e}^{-\gamma T} \mathrm{E}\left[\mathrm{e}^{-r T} \mathbf{1}\left(\tau_{u}>T\right)\right] \mathrm{d} T+\int_{0}^{\infty} \frac{R \mathrm{E}\left[\mathrm{e}^{-r \tau_{u}-\gamma T} V\left(\tau_{u}\right) \mathbf{1}\left(\tau_{u} \leq T\right)\right]}{K} \mathrm{~d} T .
\end{aligned}
$$

Using Fubini's theorem, we can rewrite (2.6) as

$$
\begin{aligned}
\hat{B}(\gamma) & =\mathrm{E}\left[\int_{0}^{\tau_{u}} \mathrm{e}^{-(\gamma+r) T} \mathrm{~d} T\right]+\mathrm{E}\left[\int_{\tau_{u}}^{\infty} \frac{R \mathrm{e}^{-r \tau_{u}-\gamma T} V\left(\tau_{u}\right) \mathbf{1}\left(\tau_{u}<\infty\right)}{K}\right] \mathrm{d} T \\
& =\frac{1-\mathrm{E}\left[\mathrm{e}^{\left.-(\gamma+r) \tau_{u}\right]}\right.}{\gamma+r}+\frac{R \mathrm{E}\left[\mathrm{e}^{-(r+\gamma) \tau_{u}} V\left(\tau_{u}\right) \mathbf{1}\left(\tau_{u}<\infty\right)\right]}{K \gamma} .
\end{aligned}
$$


Similarly, the Laplace transforms of $A_{1}(u, T)$ and $A_{2}(u, T)$ are given by

$$
\hat{A}_{1}(\gamma)=\int_{0}^{\infty} \mathrm{e}^{-\gamma T} A_{1}(u, T) \mathrm{d} T=\frac{\mathrm{E}\left[\mathrm{e}^{-(r+\gamma) \tau_{u}}\left(1-R V\left(\tau_{u}\right) / K\right) \mathbf{1}\left(\tau_{u}<\infty\right)\right]}{\gamma}
$$

and

$$
\begin{aligned}
\hat{A}_{2}(\gamma) & =\int_{0}^{\infty} \mathrm{e}^{-\gamma T} A_{2}(u, T) \mathrm{d} T \\
& =\mathrm{E}\left[\int_{0}^{\infty} \int_{t}^{\infty} \mathrm{e}^{-\gamma T-r t} \mathbf{1}\left(\tau_{u}>t\right) \mathrm{d} T \mathrm{~d} t\right] \\
& =\mathrm{E}\left[\int_{0}^{\tau_{u}} \frac{\mathrm{e}^{-(\gamma+r) t}}{\gamma} \mathrm{d} t\right] \\
& =\frac{1-\mathrm{E}\left[\mathrm{e}^{-(\gamma+r) \tau_{u}}\right]}{\gamma(\gamma+r)} .
\end{aligned}
$$

It is easy to see that the Laplace transforms in (2.7), (2.8), and (2.9) rely on the Laplace transform of $\tau_{u}$ and the expectation $\mathrm{E}\left[\mathrm{e}^{-(r+\gamma) \tau_{u}} V\left(\tau_{u}\right) \mathbf{1}\left(\tau_{u}<\infty\right)\right]$. Thus, it is helpful to derive some exact results available for the latter two.

\section{Main results}

Let $Y$ be a random variable measurable with respect to the $\sigma$-algebra $\sigma\{X(s), 0 \leq s<\infty\}$. We use the superscript $u$ in the conditional expectation $\mathrm{E}^{u}[Y]=\mathrm{E}[Y \mid X(0)=u]$ to indicate that the real-valued Markov process $\{X(t)\}$ starts from $X(0)=u$ with probability 1 . To unify the study, following Gerber and Shiu (1998), we consider the function

$$
\Phi(u)=\mathrm{E}^{u}\left[\mathrm{e}^{-\delta \tau_{u}+\xi X\left(\tau_{u}\right)} \mathbf{1}\left(\tau_{u}<\infty\right)\right] \text { for } \xi \geq 0 .
$$

Theorem 3.1. Assume that the jump size distribution $F$ is absolutely continuous. Then, the function $\Phi(u)$ is twice continuously differentiable on $(0, \infty)$ and it satisfies the integrodifferential equation

$$
\frac{1}{2} \sigma^{2} \Phi^{\prime \prime}(u)+\mu \Phi^{\prime}(u)-(\lambda+\delta) \Phi(u)=-\lambda\left[\int_{-\infty}^{-u} \mathrm{e}^{(u+z) \xi} \mathrm{d} F(z)+\int_{-u}^{\infty} \Phi(u+z) \mathrm{d} F(z)\right] .
$$

Proof. Let $u>0$. Note that the process $\{X(t)\}$ is a homogeneous strong Markov process on $(-\infty, \infty)$. The killed version of the process $\{X(t)\}$ at default time $\tau_{u}$ is

$$
\bar{X}(t)= \begin{cases}X(t) & \text { if } t<\tau_{u}, \\ \partial & \text { if } t \geq \tau_{u},\end{cases}
$$

where $\partial$ denotes the death state for the process $\{X(t)\}$. The twice killing process for the process $\{X(t)\}$ is

$$
Z(t)= \begin{cases}\bar{X}(t) & \text { if } t<\zeta \\ \partial & \text { if } t \geq \zeta\end{cases}
$$

where $\zeta$ is an exponential random variable with parameter $\delta$, independent of the process $\{X(t), t \geq 0\}$. The process $\{Z(t), t \geq 0\}$ is also a homogeneous strong Markov process in $(0, \infty)$. See, for example, Blumenthal and Getoor (1968, Chapter 3). 
Let $A$ denote the weak infinitesimal generator of the process $\{Z(t)\}$, and let $D(A)$ denote its domain. Since $\sigma>0$ in credit risk process (1.1), each function in $D(A)$ is defined on $(0, \infty)$ and is twice continuously differentiable. Using Itô's formula, we can find the infinitesimal generator

$$
\begin{aligned}
A g(u) & :=\lim _{h \downarrow 0} \frac{\mathrm{E}^{u}[g(Z(h))]-g(u)}{h} \\
& =\frac{\sigma^{2}}{2} g^{\prime \prime}(u)+\mu g^{\prime}(u)+\lambda \int_{-u}^{\infty} g(u+z) \mathrm{d} F(z)-(\delta+\lambda) g(u),
\end{aligned}
$$

where $g \in D(A)$.

Similar to Yuen et al. (2009), to prove the theorem, it is sufficient to show that the function $\Phi(u)$ on $(0, \infty)$ is in $D(A)$. To show this, from Dynkin (1965, Chapter 1), we need to only prove that $\lim _{h \downarrow 0} \mathrm{E}^{u}[\Phi(Z(h))]=\Phi(u)$ for each $u \in(0, \infty)$ and that, as a function of variable $u$ on $(0, \infty)$, the limit

$$
\lim _{h \downarrow 0} \frac{E^{u}[\Phi(Z(h))]-\Phi(u)}{h}
$$

is bounded and continuous.

For $t \geq 0$, let $\theta_{t}$ denote the shift operator from $\Omega$ to itself defined by $X(s, \omega) \circ \theta_{t}=$ $X\left(s, \theta_{t} \omega\right)=X(s+t, \omega)$. We refer the reader to Revuz and Yor $(1991, \mathrm{pp} .34,97)$ for a detailed definition.

It is easy to see that

$$
\begin{aligned}
\mathrm{E}^{u}[\Phi(Z(h))] & =\mathrm{E}^{u}\left[\mathbf{1}\left(h<\tau_{u}<\infty\right) \mathbf{1}(\zeta>h) \Phi(X(h))\right] \\
& =\mathrm{e}^{-\delta h} \mathrm{E}^{u}\left[\mathbf{1}\left(h<\tau_{u}<\infty\right) \Phi(X(h))\right],
\end{aligned}
$$

where, by convention, $\Phi(\partial)=0$. Conditioning on $\left\{h<\tau_{u}<\infty\right\}$, we have $\tau_{u}=h+\tau_{u} \circ \theta_{h}$. A standard use of the homogeneous Markov property of the process $X(t)$ gives

$$
\begin{aligned}
\mathrm{E}^{u}\left[\mathbf{1}\left(h<\tau_{u}<\infty\right) \Phi(X(h))\right] & =\mathrm{E}^{u}\left[\mathbf{1}\left(h<\tau_{u}<\infty\right) \mathrm{E}^{u}\left[\mathrm{e}^{-\delta \tau_{u}+\xi X\left(\tau_{u}\right)} \circ \theta_{h} \mid \Im_{h}\right]\right] \\
& =\mathrm{E}^{u}\left[\mathrm{E}^{u}\left[\mathbf{1}\left(h<\tau_{u}<\infty\right) \mathrm{e}^{-\delta \tau_{u}+\xi X\left(\tau_{u}\right)} \circ \theta_{h} \mid \Im_{h}\right]\right] \\
& =\mathrm{E}^{u}\left[\mathbf{1}\left(h<\tau_{u}<\infty\right) \mathrm{e}^{-\delta \tau_{u} \circ \theta_{h}+\xi X\left(\tau_{u} \circ \theta_{h}+h\right)}\right] \\
& =\mathrm{E}^{u}\left[\mathbf{1}\left(h<\tau_{u}<\infty\right) \mathrm{e}^{\delta h-\delta \tau_{u}+\xi X\left(\tau_{u}\right)}\right] \\
& =\mathrm{e}^{\delta h} \Phi(u)-\mathrm{e}^{\delta h} \mathrm{E}^{u}\left[\mathbf{1}\left(\tau_{u} \leq h\right) \mathrm{e}^{-\delta \tau_{u}+\xi X\left(\tau_{u}\right)}\right] .
\end{aligned}
$$

Hence, from (3.3) and (3.4) we obtain

$$
\mathrm{E}^{u}[\Phi(Z(h))]=\Phi(u)-\mathrm{E}^{u}\left[\mathbf{1}\left(\tau_{u} \leq h\right) \mathrm{e}^{-\delta \tau_{u}+\xi X\left(\tau_{u}\right)}\right] .
$$

Equation (3.5) implies that

$$
\lim _{h \downarrow 0} \frac{\mathrm{E}^{u}[\Phi(Z(h))]-\Phi(u)}{h}=-\lim _{h \downarrow 0} \frac{\mathrm{E}^{u}\left[\mathbf{1}\left(\tau_{u} \leq h\right) \mathrm{e}^{-\delta \tau_{u}+\xi X\left(\tau_{u}\right)}\right]}{h} .
$$

Note that $\mathrm{P}(N(h) \geq 2)=o(h)$ and $\mathrm{P}\left(\inf _{0 \leq s \leq h}(\mu s+\sigma W(s))+u \leq 0\right)=o(h)$ for each $u>0$. Given $N(h)=1$, let $\epsilon(h)$ denote the jump time of the Poisson process $\{N(t)\}$ in $[0, h]$. 
Conditioning on the number of the jump times of the Poisson process $\{N(t)\}$ in $[0, h]$ and using the independence assumptions, we obtain

$$
\begin{aligned}
& \lim _{h \downarrow 0} \frac{\mathrm{E}^{u}\left[\mathbf{1}\left(\tau_{u} \leq h\right) \mathrm{e}^{-\delta \tau_{u}+\xi X\left(\tau_{u}\right)}\right]}{h} \\
&=\lim _{h \downarrow 0} \frac{1}{h}\left[\mathrm{E}^{u}\left[\mathbf{1}\left(\tau_{u} \leq h\right) \mathbf{1}(N(h)=0) \mathrm{e}^{-\delta \tau_{u}+\xi X\left(\tau_{u}\right)}\right]\right. \\
& \quad+\mathrm{E}^{u}\left[\mathbf{1}\left(\tau_{u} \leq h\right) \mathbf{1}(N(h)=1) \mathrm{e}^{-\delta \tau_{u}+\xi X\left(\tau_{u}\right)}\right] \\
&\left.\quad+\mathrm{E}^{u}\left[\mathbf{1}\left(\tau_{u} \leq h\right) \mathbf{1}(N(h) \geq 2) \mathrm{e}^{-\delta \tau_{u}+\xi X\left(\tau_{u}\right)}\right]\right] \\
&=\lim _{h \downarrow 0} \frac{1}{h}\left[\mathrm{e}^{-\lambda h} \mathrm{E}^{u}\left[\mathbf{1}\left(\inf _{0 \leq s \leq h}(\mu s+\sigma W(s))+u \leq 0\right) \mathbf{1}\left(\tau_{u} \leq h\right) \mathrm{e}^{-\delta \tau_{u}+\xi X\left(\tau_{u}\right)}\right]\right. \\
& \quad+\lambda h \mathrm{e}^{-\lambda h} \mathrm{E}^{u}\left[\mathbf{1}\left(\inf _{0 \leq s \leq h}\left(\mu s+\sigma W(s)+\mathbf{1}(s \geq \epsilon(h)) Z_{1}\right)+u \leq 0\right)\right. \\
&\left.\left.\quad \times \mathrm{e}^{-\delta \epsilon(h)+\xi X(\epsilon(h))}\right]+o(h)\right] \\
&=\lim _{h \downarrow 0} \lambda \mathrm{e}^{-\lambda h} \mathrm{E}^{u}\left[\mathbf{1}\left(\inf _{0 \leq s \leq h}\left(\mu s+\sigma W(s)+\mathbf{1}(s \geq \epsilon(h)) Z_{1}\right)+u \leq 0\right) \mathrm{e}^{-\delta \epsilon(h)+\xi X(\epsilon(h))}\right] \\
&=\lambda \int_{-\infty}^{-u} \mathrm{e}^{(u+z) \xi} \mathrm{d} F(z) .
\end{aligned}
$$

Therefore, we have

$$
\lim _{h \downarrow 0} \frac{\mathrm{E}^{u}[\Phi(Z(h))]-\Phi(u)}{h}=-\lambda \int_{-\infty}^{-u} \mathrm{e}^{(u+z) \xi} \mathrm{d} F(z) .
$$

By assumption, $F$ is absolutely continuous. Hence, the integral $\int_{-\infty}^{-u} \mathrm{e}^{(u+z) \xi} \mathrm{d} F(z)$ is bounded and continuous in $u$ on $(0, \infty)$, given $\xi \geq 0$. This means that $\lim _{h \downarrow 0} \mathrm{E}^{u}[\Phi(Z(h))]=\Phi(u)$ for each $u \in(0, \infty)$. Therefore, the function $\Phi(u)$ on $(0, \infty)$ is in $D(A)$ and, hence, it is twice continuously differentiable on $(0, \infty)$. Replacing the function $g$ by the function $\Phi$ in (3.2) and then using (3.6), we obtain (3.1). This completes the proof.

It is easy to see that $L(u)=\left.\Phi(u)\right|_{\xi=0}$ and $\phi(u)=\left.K \Phi(u)\right|_{\xi=1}$ on $(0, \infty)$. Hence, we have the following two corollaries.

Corollary 3.1. Assume that the jump size distribution $F$ is absolutely continuous. Then, the Laplace transform $L(u)$ is twice continuously differentiable on $(0, \infty)$ and it satisfies the integro-differential equation

$$
\frac{1}{2} \sigma^{2} L^{\prime \prime}(u)+\mu L^{\prime}(u)-(\lambda+\delta) L(u)=-\lambda\left[F(-u)+\int_{-u}^{\infty} L(u+z) \mathrm{d} F(z)\right], \quad u>0 .
$$

Corollary 3.2. Assume that the jump size distribution $F$ is absolutely continuous. Then, the expected present market value $\phi(u)$ at the default time is twice continuously differentiable on $(0, \infty)$ and it satisfies the integro-differential equation

$$
\frac{1}{2} \sigma^{2} \phi^{\prime \prime}(u)+\mu \phi^{\prime}(u)-(\lambda+\delta) \phi(u)=-\lambda\left[\int_{-\infty}^{-u} K \mathrm{e}^{u+z} \mathrm{~d} F(z)+\int_{-u}^{\infty} \phi(u+z) \mathrm{d} F(z)\right] .
$$


Remark 3.1. Denote by $\mu_{Z}$ the mean of $Z_{i}$. Assume that $\bar{\mu}=\mu+\lambda \mu_{Z}>0$, so that we have $V(t) \rightarrow \infty$ as $t \rightarrow \infty$ and $\lim _{u \rightarrow \infty} \tau_{u}=\infty$ almost surely. In this case, we have

$$
\lim _{u \rightarrow \infty} L(u)=0 .
$$

Thus, we could obtain the Laplace transform $L(u)$ by solving the boundary value problems (2.1), (3.7), and (3.9). In the case where $\bar{\mu}<0$, the boundary condition (3.9) does not hold any more and we cannot in general obtain the Laplace transform $L(u)$ by solving only the boundary value problems (2.1) and (3.7). However, using the boundedness of the Laplace transform and by solving the boundary value problems (2.1) and (3.7), we can obtain a closed-form expression for the Laplace transform $L(u)$ when the jump size distribution $F$ is a hyperexponential distribution. Similar arguments also hold for the function $\phi(u)$.

Example 3.1. We now assume that the jump size distribution $F$ is a hyperexponential distribution, that is, its density function, denoted by $f$, can be expressed as

$$
f(z)= \begin{cases}p_{1} \alpha_{1} \mathrm{e}^{-\alpha_{1} z}+p_{2} \alpha_{2} \mathrm{e}^{-\alpha_{2} z}+\cdots+p_{n} \alpha_{n} \mathrm{e}^{-\alpha_{n} z}, & z \geq 0, \\ q_{1} \beta_{1} \mathrm{e}^{\beta_{1} z}+q_{2} \beta_{2} \mathrm{e}^{\beta_{2} z}+\cdots+q_{n} \beta_{n} \mathrm{e}^{\beta_{n} z}, & z<0,\end{cases}
$$

with $0<\alpha_{1}<\alpha_{2}<\cdots<\alpha_{n}, 0<\beta_{1}<\beta_{2}<\cdots<\beta_{n}, 0 \leq p_{i}, q_{i} \leq 1, i=1, \ldots, n$, and $\sum_{i=1}^{n}\left(p_{i}+q_{i}\right)=1$.

Though the density function $f(z)$ given in (3.10) may not be continuous at $z=0$, the hyperexponential distribution $F$ is absolutely continuous. Hence, by Theorem 3.1, the corresponding Laplace transform $L(u)$ in this example is twice continuously differentiable on $(0, \infty)$ and it satisfies (3.7).

Let $\mathbb{I}$ denote the identity operator, and let $\mathbb{D}$ denote the differential operator. Define the differential operator polynomial

$$
h_{2}(\mathbb{D})=\frac{1}{2} \sigma^{2} \mathbb{D}^{2}+\mu \mathbb{D}-(\lambda+\delta) \mathbb{I},
$$

where, by convention, $\mathbb{D}^{2} L(u)=\mathbb{D}(\mathbb{D} L(u))$. Similarly, we define $h_{2}(x)=\frac{1}{2} \sigma^{2} x^{2}+\mu x-$ $(\lambda+\delta)$. Inserting (3.10) into (3.7) gives

$$
\begin{aligned}
h_{2}(\mathbb{D}) L(u)=-\lambda & {\left[\sum_{i=1}^{n} q_{i} \mathrm{e}^{-\beta_{i} u}+\int_{0}^{u} L(s) \sum_{i=1}^{n} q_{i} \beta_{i} \mathrm{e}^{\beta_{i}(s-u)} \mathrm{d} s\right.} \\
& \left.+\int_{u}^{\infty} L(s) \sum_{i=1}^{n} p_{i} \alpha_{i} \mathrm{e}^{-\alpha_{i}(s-u)} \mathrm{d} s\right] .
\end{aligned}
$$

Similar to Gerber and Shiu (2005) and Wang and Wu (2008), applying the differential operator polynomial $\prod_{i=1}^{n}\left[\left(\mathbb{D}+\beta_{i} \mathbb{I}\right)\left(\mathbb{D}-\alpha_{i} \mathbb{I}\right)\right]$ to the both sides of (3.11) yields the differential equation

$$
\begin{aligned}
\left(\prod_{i=1}^{n}\left[\left(\mathbb{D}+\beta_{i} \mathbb{I}\right)\left(\mathbb{D}-\alpha_{i} \mathbb{I}\right)\right]\right) h_{2}(\mathbb{D}) L(u), \\
=\lambda \sum_{i=1}^{n} p_{i} \alpha_{i}\left(\left(\mathbb{D}+\beta_{i} \mathbb{I}\right) \prod_{j=1, j \neq i}^{n}\left[\left(\mathbb{D}+\beta_{j} \mathbb{I}\right)\left(\mathbb{D}-\alpha_{j} \mathbb{I}\right)\right]\right) L(u) \\
\quad-\lambda \sum_{i=1}^{n} q_{i} \beta_{i}\left(\left(\mathbb{D}-\alpha_{i} \mathbb{I}\right) \prod_{j=1, j \neq i}^{n}\left[\left(\mathbb{D}+\beta_{j} \mathbb{I}\right)\left(\mathbb{D}-\alpha_{j} \mathbb{I}\right)\right]\right) L(u) .
\end{aligned}
$$




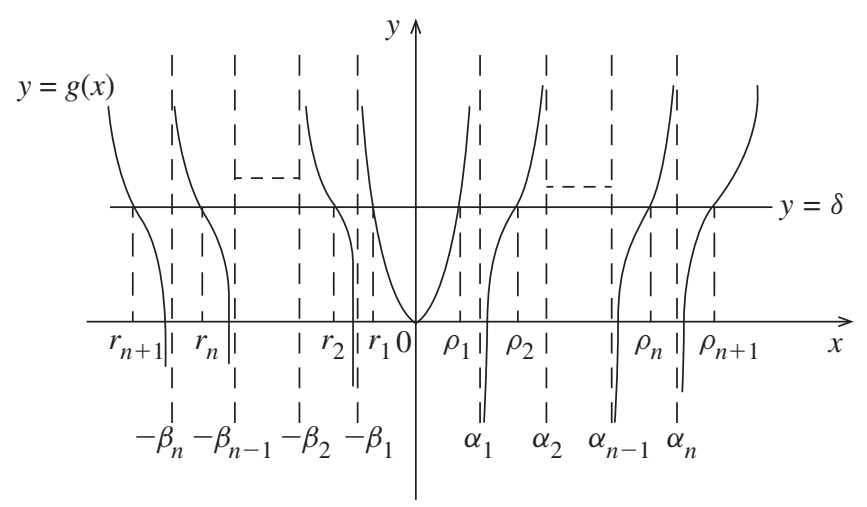

Figure 1: The roots of (3.15).

The characteristic equation of (3.12) is

$$
\begin{aligned}
& \left(\prod_{i=1}^{n}\left[\left(x+\beta_{i}\right)\left(x-\alpha_{i}\right)\right]\right) h_{2}(x) \\
& \quad=\lambda \sum_{i=1}^{n} p_{i} \alpha_{i} \prod_{j=1}^{n} \frac{\left(x+\beta_{j}\right)\left(x-\alpha_{j}\right)}{x-\alpha_{i}}-\lambda \sum_{i=1}^{n} q_{i} \beta_{i} \prod_{j=1}^{n} \frac{\left(x+\beta_{j}\right)\left(x-\alpha_{j}\right)}{x+\beta_{i}} .
\end{aligned}
$$

By partial fraction decomposition we can rewrite (3.13) as

$$
h_{2}(x)=\lambda \sum_{i=1}^{n}\left(\frac{p_{i} \alpha_{i}}{x-\alpha_{i}}-\frac{q_{i} \beta_{i}}{x+\beta_{i}}\right) .
$$

Define

$$
g(x)=\frac{1}{2} \sigma^{2} x^{2}+\mu x-\lambda+\lambda \sum_{i=1}^{n}\left(\frac{q_{i} \beta_{i}}{x+\beta_{i}}-\frac{p_{i} \alpha_{i}}{x-\alpha_{i}}\right) .
$$

Then, (3.14) can be rewritten as

$$
g(x)=\delta .
$$

Note that (3.13) has $2 n+2$ roots and it has the same roots as (3.15). We can verify that (3.15) has $2 n+2$ distinct roots $r_{i}, \rho_{i}, i=1,2, \ldots, n+1$, with

$$
\begin{gathered}
r_{n+1}<-\beta_{n}<r_{n}<-\beta_{n-1}<\cdots<-\beta_{1}<r_{1}<0, \\
0<\rho_{1}<\alpha_{1}<\rho_{2}<\alpha_{2}<\cdots<\alpha_{n}<\rho_{n+1}
\end{gathered}
$$

(see Figure 1). Hence, the Laplace transform $L(u)$ has the form

$$
L(u)=\sum_{i=1}^{n+1} c_{i} \mathrm{e}^{r_{i} u}+\sum_{i=1}^{n+1} d_{i} \mathrm{e}^{\rho_{i} u}, \quad u \geq 0,
$$

where the $c_{i} \mathrm{~s}$ and $d_{i} \mathrm{~s}$ are arbitrary constants. From the boundedness of the Laplace transform and boundary condition $(2.1)$, we see that

$$
d_{i}=0, \quad i=1, \ldots, n+1,
$$


and

$$
\sum_{i=1}^{n+1} c_{i}=1
$$

It follows that

$$
L(u)=\sum_{i=1}^{n+1} c_{i} \mathrm{e}^{r_{i} u}, \quad u \geq 0 .
$$

Substituting $L(u)$ given in (3.17) into (3.11) and equating the coefficients of $\mathrm{e}^{-\beta_{i} u}$ yields

$$
\sum_{j=1}^{n+1} \frac{c_{j} \beta_{i}}{\beta_{i}+r_{j}}=1, \quad i=1, \ldots, n .
$$

Let $\boldsymbol{A}$ denote the $(n+1) \times(n+1)$ coefficient matrix of the linear system (3.16) and (3.18). We have

$$
\boldsymbol{A}=\left(\begin{array}{ccc}
1 & \cdots & 1 \\
\frac{\beta_{1}}{\beta_{1}+r_{1}} & \cdots & \frac{\beta_{1}}{\beta_{1}+r_{n+1}} \\
\vdots & \ddots & \vdots \\
\frac{\beta_{n}}{\beta_{n}+r_{1}} & \cdots & \frac{\beta_{n}}{\beta_{n}+r_{n+1}}
\end{array}\right) .
$$

Denote the determinant of the matrix $\boldsymbol{A}$ by $\operatorname{det} \boldsymbol{A}$. We can verify that $\operatorname{det} \boldsymbol{A} \neq 0$. Let $\boldsymbol{A}_{k}$ denote the matrix obtained from $\boldsymbol{A}$ by replacing its $k$ th column by the $(n+1)$-dimensional column vector $\boldsymbol{W}=(1,1, \ldots, 1)^{\top}, k=1,2, \ldots, n+1$. Thus, we have

$$
c_{k}=(\operatorname{det} \boldsymbol{A})^{-1} \operatorname{det} \boldsymbol{A}_{k}, \quad k=1,2, \ldots, n+1 .
$$

Hence, (3.17) and (3.19) give the closed-form expression for the Laplace transform $L(u)$.

For $n=2$, we have

$$
L(u)=c_{1} \mathrm{e}^{r_{1} u}+c_{2} \mathrm{e}^{r_{2} u}+c_{3} \mathrm{e}^{r_{3} u},
$$

where $r_{1}, r_{2}$, and $r_{3}$ with $r_{3}<-\beta_{2}<r_{2}<-\beta_{1}<r_{1}<0$ are the three roots of the equation

$$
h_{2}(x)+\lambda\left(\frac{q_{1} \beta_{1}}{x+\beta_{1}}+\frac{q_{2} \beta_{2}}{x+\beta_{2}}+\frac{p_{1} \alpha_{1}}{\alpha_{1}-x}+\frac{p_{2} \alpha_{2}}{\alpha_{2}-x}\right)=0,
$$

and

$$
\begin{aligned}
& c_{1}=\frac{r_{2} r_{3}\left(r_{1}+\beta_{1}\right)\left(r_{1}+\beta_{2}\right)}{\left(r_{1}-r_{2}\right)\left(r_{1}-r_{3}\right) \beta_{1} \beta_{2}}, \\
& c_{2}=\frac{r_{1} r_{3}\left(r_{2}+\beta_{1}\right)\left(r_{2}+\beta_{2}\right)}{\left(r_{2}-r_{1}\right)\left(r_{2}-r_{3}\right) \beta_{1} \beta_{2}}, \\
& c_{3}=\frac{r_{1} r_{2}\left(r_{3}+\beta_{1}\right)\left(r_{3}+\beta_{2}\right)}{\left(r_{3}-r_{1}\right)\left(r_{3}-r_{2}\right) \beta_{1} \beta_{2}} .
\end{aligned}
$$

Similarly, for $n=1$, we have

$$
L(u)=c_{1} \mathrm{e}^{r_{1} u}+c_{2} \mathrm{e}^{r_{2} u},
$$


where $r_{1}$ and $r_{2}$ with $r_{2}<-\beta_{1}<r_{1}<0$ are the two roots of the equation

$$
h_{2}(x)+\lambda\left(\frac{q_{1} \beta_{1}}{x+\beta_{1}}+\frac{p_{1} \alpha_{1}}{\alpha_{1}-x}\right)=0
$$

and

$$
c_{1}=\frac{r_{2}\left(r_{1}+\beta_{1}\right)}{\beta_{1}\left(r_{2}-r_{1}\right)}, \quad c_{2}=\frac{r_{1}\left(r_{2}+\beta_{1}\right)}{\beta_{1}\left(r_{1}-r_{2}\right)} .
$$

Example 3.2. Under the assumptions of Example 3.1, we can obtain a closed-form expression for $\phi(u)$ by solving (3.8) with boundary conditions (2.2).

Using boundary conditions (2.2) and following the same arguments as used to derive (3.17) and (3.19), we obtain

$$
\phi(u)=\sum_{i=1}^{n+1} K \eta_{i} \mathrm{e}^{r_{i} u}
$$

where the $\eta_{i}$ s solve the system

$$
A \eta=Q
$$

with

$$
\boldsymbol{\eta}=\left(\eta_{1}, \ldots, \eta_{n+1}\right)^{\top} \quad \text { and } \quad \boldsymbol{Q}=\left(1, \frac{\beta_{1}}{\left(\beta_{1}+1\right)}, \ldots, \frac{\beta_{n}}{\left(\beta_{n}+1\right)}\right)^{\top}
$$

being two $(n+1)$-dimensional column vectors, and the $r_{i}$ s are the roots of (3.14).

For $n=2$, we have

$$
\phi(u)=K\left(\eta_{1} \mathrm{e}^{r_{1} u}+\eta_{2} \mathrm{e}^{r_{2} u}+\eta_{3} \mathrm{e}^{r_{3} u}\right),
$$

where $r_{1}, r_{2}$, and $r_{3}$ are the three roots of (3.20), and

$$
\begin{aligned}
\eta_{1}= & \frac{\left(r_{1}+\beta_{1}\right)\left(r_{1}+\beta_{2}\right)\left(r_{2}-1\right)\left(r_{3}-1\right)}{\left(r_{1}-r_{2}\right)\left(r_{1}-r_{3}\right)\left(1+\beta_{1}\right)\left(1+\beta_{2}\right)}, \\
\eta_{2}= & \frac{\left(r_{2}+\beta_{1}\right)\left(r_{2}+\beta_{2}\right)\left(r_{1}-1\right)\left(r_{3}-1\right)}{\left(r_{2}-r_{1}\right)\left(r_{2}-r_{3}\right)\left(1+\beta_{1}\right)\left(1+\beta_{2}\right)}, \\
\eta_{3}= & \frac{\left(r_{3}+\beta_{1}\right)\left(r_{3}+\beta_{2}\right)\left(r_{1}-1\right)\left(r_{2}-1\right)}{\left(r_{3}-r_{1}\right)\left(r_{3}-r_{2}\right)\left(1+\beta_{1}\right)\left(1+\beta_{2}\right)} .
\end{aligned}
$$

Similarly, for $n=1$, we have

$$
\phi(u)=K\left(\eta_{1} \mathrm{e}^{r_{1} u}+\eta_{2} \mathrm{e}^{r_{2} u}\right),
$$

where $r_{1}$ and $r_{2}$ are the two roots of (3.22), and

$$
\eta_{1}=\frac{\left(r_{1}+\beta_{1}\right)\left(1-r_{2}\right)}{\left(r_{1}-r_{2}\right)\left(1+\beta_{1}\right)}, \quad \eta_{2}=\frac{\left(r_{2}+\beta_{1}\right)\left(r_{1}-1\right)}{\left(r_{1}-r_{2}\right)\left(1+\beta_{1}\right)} .
$$

\section{Numerical solutions}

In this section we present some numerical results on the fair premium rate of the zero-coupon bond, the default probability, and the credit spread when the jumps have a hyperexponential distribution. We first introduce the Gaver-Stehfest algorithm for inverting the Laplace transform 
which was used in Kou and Wang (2003). For a nonnegative function $f(t)$, we denote by $\hat{F}$ its Laplace transform, that is,

$$
\hat{F}(\delta)=\int_{0}^{\infty} \mathrm{e}^{-\delta t} f(t) \mathrm{d} t,
$$

where $\delta>0$. Using (4.1), and Equations (5.1) and (5.2) of Kou and Wang (2003), we obtain

$$
f(t)=\lim _{m \rightarrow \infty} \sum_{k=1}^{m} \varpi(k, m) \bar{F}_{k}(t),
$$

where

$$
\begin{gathered}
\varpi(k, m)=(-1)^{m-k} \frac{k^{m}}{k !(m-k) !}, \\
\bar{F}_{m}(t)=\frac{\ln (2)}{t} \frac{(2 m) !}{m !(m-1) !} \sum_{k=0}^{m}(-1)^{k}\left(\begin{array}{l}
m \\
k
\end{array}\right) \hat{F}\left((m+k) \frac{\ln (2)}{t}\right),
\end{gathered}
$$

and $m$ is a positive integer. As pointed out in Section 5 of Kou and Wang (2003), we can increase the numerical stability by replacing $\bar{F}_{k}(t)$ in (4.2) by $\bar{F}_{k+2}(t)$, that is, we can use the approximation formula

$$
f(t) \simeq \sum_{k=1}^{m} \varpi(k, m) \bar{F}_{k+2}(t)
$$

where $\varpi$ and $\bar{F}$ are given by (4.3) and (4.4), respectively. The algorithm quickly converges and it typically converges nicely even for $m$ between 5 and 10 .

Let $\hat{\psi}(u, \delta)$ denote the Laplace transform of $\Psi(u, t)=\mathrm{P}\left(\tau_{u} \leq t\right)$. Similar to Kou and Wang (2003), we have

$$
\hat{\psi}(u, \delta)=\int_{0}^{\infty} \mathrm{e}^{-\delta t} \mathrm{P}\left(\tau_{u} \leq t\right) \mathrm{d} t=\frac{1}{\delta} \int_{0}^{\infty} \mathrm{e}^{-\delta t} \mathrm{dP}\left(\tau_{u} \leq t\right)=\frac{1}{\delta} L(u) .
$$

For simplicity, we choose $n=2$ in Example 3.1. Thus, from (3.17) we have

$$
\hat{\psi}(u, \delta)=\frac{1}{\delta}\left(c_{1} \mathrm{e}^{r_{1} u}+c_{2} \mathrm{e}^{r_{2} u}+c_{3} \mathrm{e}^{r_{3} u}\right),
$$

where $c_{1}, c_{2}$, and $c_{3}$ are given in (3.21a)-(3.21c), and $r_{1}, r_{2}$, and $r_{3}$ are the roots of (3.20) with $\delta$ being replaced by $\gamma+r$ in $h(x)$. Similarly, from (3.17) and (3.23), we obtain

$$
\begin{aligned}
& \hat{B}(\gamma)=\frac{1}{\gamma+r}+\left(\frac{R \eta_{1}}{\gamma}-\frac{c_{1}}{\gamma+r}\right) \mathrm{e}^{r_{1} u}+\left(\frac{R \eta_{2}}{\gamma}-\frac{c_{2}}{\gamma+r}\right) \mathrm{e}^{r_{2} u}+\left(\frac{R \eta_{3}}{\gamma}-\frac{c_{3}}{\gamma+r}\right) \mathrm{e}^{r_{3} u} \\
& \hat{A}_{1}(\gamma)=\frac{\left(c_{1}-R \eta_{1}\right) \mathrm{e}^{r_{1} u}+\left(c_{2}-R \eta_{2}\right) \mathrm{e}^{r_{2} u}+\left(c_{1}-R \eta_{3}\right) \mathrm{e}^{r_{3} u}}{\gamma} \\
& \hat{A}_{2}(\gamma)=\frac{1-c_{1} \mathrm{e}^{r_{1} u}-c_{2} \mathrm{e}^{r_{2} u}-c_{3} \mathrm{e}^{r_{3} u}}{\gamma(\gamma+r)}
\end{aligned}
$$

where $\eta_{1}, \eta_{2}$, and $\eta_{3}$ are given in (3.24a)-(3.24c). 
It is easy to see that

$$
\operatorname{var}(X(t))=\left[\sigma^{2}+2 \lambda\left(\frac{p_{1}}{\alpha_{1}^{2}}+\frac{p_{2}}{\alpha_{2}^{2}}+\frac{q_{1}}{\beta_{1}^{2}}+\frac{q_{2}}{\beta_{2}^{2}}\right)\right] t=: \sigma_{X}^{2} t .
$$

To investigate the impact of jumps on the default probability, the fare premium, and the credit spread, following Zhou (2001) and Le Courtois and Quittard-Pinon (2006), we keep $\sigma_{X}^{2}$ constant, so that the variations in the default probability, the fare premium, and the credit spread are mainly caused by the jump component rather than by the changes of the whole value of $\sigma_{X}^{2}$. Fix the parameters $r=0.03, R=0.8, p_{1}=p_{2}=q_{1}=q_{2}=\frac{1}{4}, \mu=0.02, \sigma_{X}=0.2, V_{0} / K=2$, and, hence, $u=0.7$. A purely diffusion process corresponds to $\lambda=0$ and, therefore, $\sigma_{X}=$ $\sigma=0.2$. For jump size parameters, we consider two different cases.

Case A. $\alpha_{1}=\beta_{1}=5, \alpha_{2}=\beta_{2}=10$,

Case B. $\alpha_{1}=\beta_{1}=10, \alpha_{2}=\beta_{2}=20$.

It is obvious that the variation of the jump size in case A is larger than that in case B.

We choose $m=9$ in (4.5). Applying (2.4), (4.5), and (4.6)-(4.9), we obtain numerical solutions for the default probability, the fair premium, and the credit spread. Some numerical results for the impact of jumps on the default probability are shown in Figure 2. As can be seen from the figure the defaults are caused mainly by jumps rather than by the diffusion part over a relatively short interval of time at the beginning. Then, the diffusion part contributes more to the default probability than the jump for relatively larger times $t$. We can also see that the default probability of credit risk process (1.1) with jumps is larger than that with no jumps over a short interval at the beginning. This fact was also noted in Zhou (2001), Le Courtois and Quittard-Pinon (2006), and Ramezani and Zeng (2007).

Figure 2(a) shows that the default probability is an increasing function of the jump intensity $\lambda$ over a short interval at the beginning and a decreasing function of $\lambda$ for relatively larger times $t$. This is because increasing the value of $\lambda$ means diminishing $\sigma$, owing to the facts that $\sigma_{X}$ is assumed to be a constant and a higher value of $\lambda$ corresponds to a smaller $\sigma$ for fixed jump sizes.

Given the jump intensity, Figure 2(b) shows that a firm with larger jump sizes (case A) is more likely to default over a relatively short interval of time at the beginning and the reverse relationship holds for relatively large times $t$.

In Figure 2(c), we assumed that the variation of the compound Poisson process in process (1.1) for case A is equal to that for case B. From Figure 2(c) we see that the default probability with larger intensity value $\lambda$ and smaller jump sizes is closer to that for a purely diffusive process. This is intuitively clear because the compound Poisson process in process (1.1) for case $\mathrm{A}$ is closer to a purely diffusive process than for case $\mathrm{B}$.

In Figure 3 we present the influence of jumps on the credit spread. From Figure 3(a) we see that a larger variation of jump size corresponds to a higher value of credit spread over a short interval at the beginning, given the intensity $\lambda$.

For a given jump size, Figure 3(b) indicates that the credit spread increases in intensity $\lambda$ over a short interval at the beginning.

In Figure 4 we present the influence of jumps on the fair premium of the zero-coupon bond. From Figure 4(a) we see that a jump size with larger variation corresponds to a higher fair premium rate, given the intensity $\lambda$. For the same jump size, Figure 4(b) shows that the fair premium rate is an increasing function of the intensity $\lambda$. 


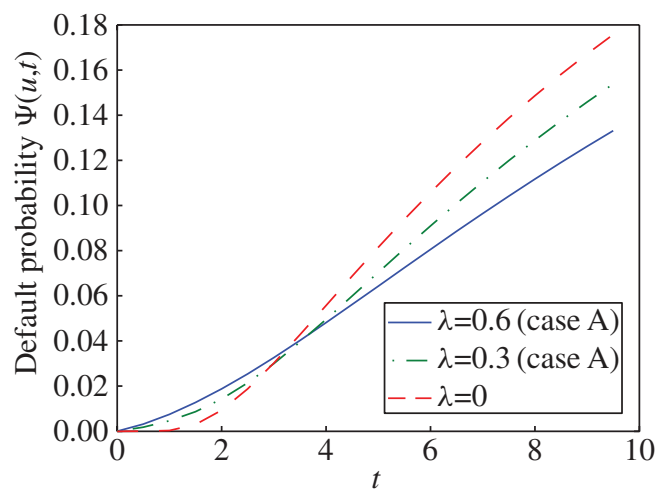

(a)

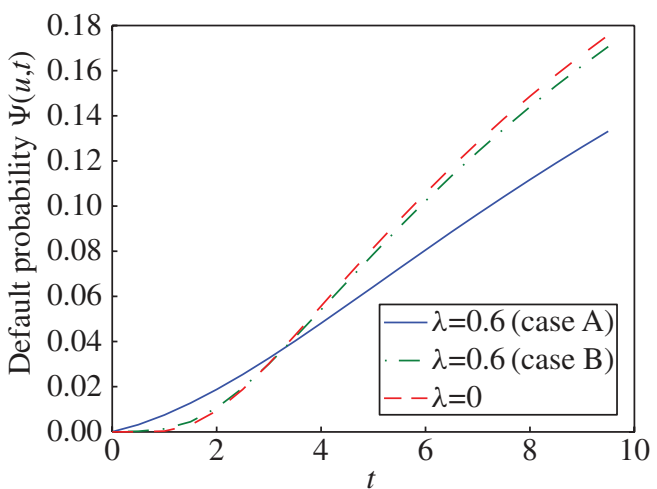

(b)

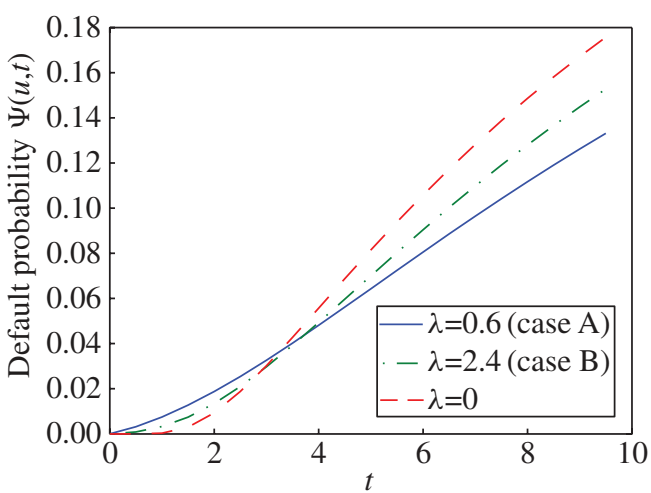

(c)

FIGURE 2: Relations between the default probability and (a) the jump intensity, (b) the variation of the jump size, and (c) the total jump component.

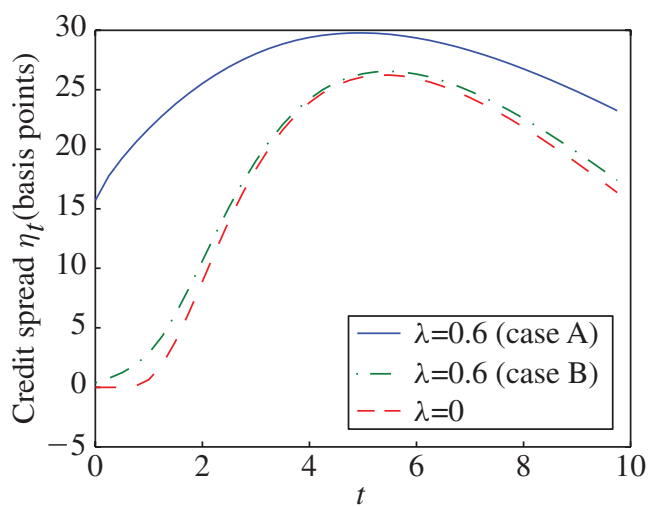

(a)

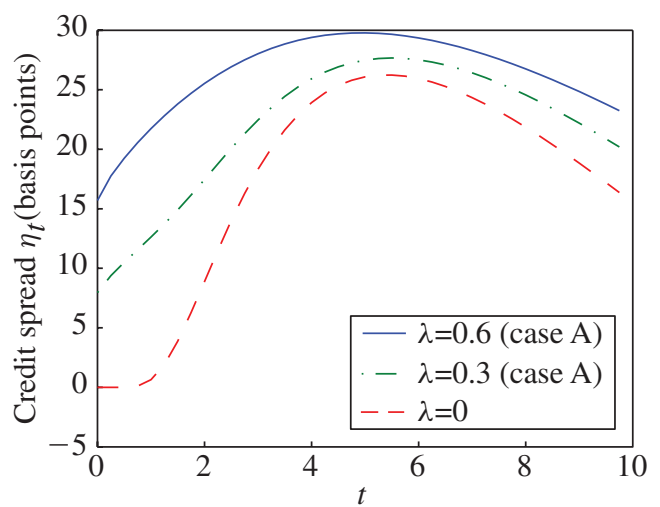

(b)

FIgURE 3: Relations between the credit spread and (a) the jump size and (b) the jump intensity. 


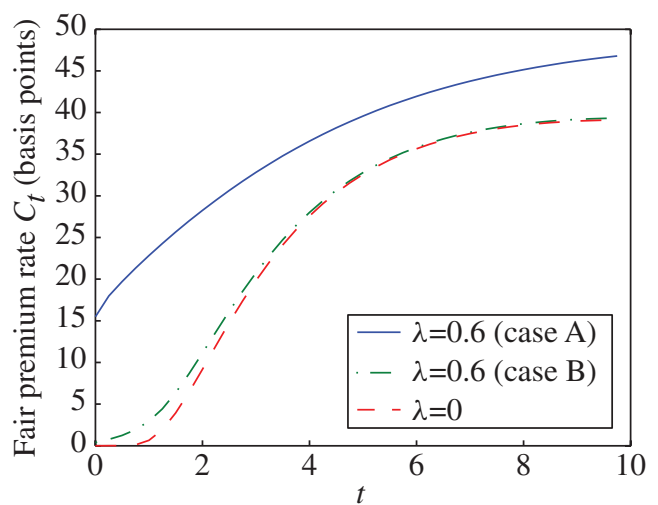

(a)

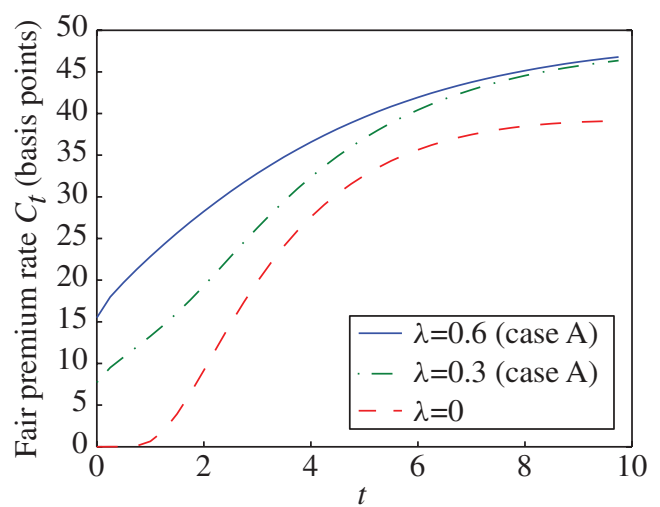

(b)

FiguRE 4: Relations between the fair premium rate and (a) the variation of the jump size and (b) the jump intensity.

\section{Conclusions}

In this paper we have considered a structural form credit risk model with jumps and provided ways to value the price and the fair premium of the zero-coupon bond. Both the price and the fair premium of the zero-coupon bond rely on the Laplace transforms of the default time and on the firm's expected present market value at default. We derived sufficient conditions under which the firm's expected present market value at default is twice continuously differentiable using a probabilistic method. Closed-form expressions for the Laplace transform of the default time and for the firm's expected present market value at default were obtained by solving certain integro-differential equations when the jumps have a hyperexponential distribution. We presented numerical illustrations to show how the jumps in the model impact on the default probability, the fair premium, and the credit spread.

Since the hyperexponential distribution is rich enough to approximate many other distributions, including some heavy-tailed distributions, in the sense of weak convergence, we may use credit risk process (1.1) with jumps having a hyperexponential distribution to approximate some models with jumps that are generally two-sided distributed when evaluating the credit risk for the proposed model. The jump magnitudes in credit risk process (1.1) need not be hyperexponentially distributed. Hence, another important topic for further research is the pricing of the zero-coupon bond under a general jump size distribution.

\section{Acknowledgements}

The authors thank the anonymous referee for helpful comments to improve an earlier version of the paper. The research of Yinghui Dong was supported by the Graduate Innovation Program (CX09B-017Z) of Jiangsu Province of China and the Natural Science Foundation for institutions (10KJB110010) of Jiangsu Province of China. The research of Guojing Wang was supported by the Natural Science Foundation (KB2008155) of Jiangsu Province of China and the Research Fund for the Doctorial Program of Higher Education. The research of Rong Wu was supported by the National Basic Research Program (973 Program: 2007CB814905) of China and the Natural Science Foundation (10871102) of China. 


\section{References}

BLACK, F. And Cox, J. (1976). Valuing corporate securities liabilities: some effects of bond indenture provisions. J. Finance 31, 351-367.

Black, F. And Scholes, M. (1973). The pricing of options and corporate liabilities. J. Political Economy 81, $637-654$.

Blumenthal, R. M. and Getoor, R. K. (1968). Markov Processes and Potential Theory. Academic Press, New York.

Chen, C.-J. And Panjer, H. (2009). A bridge from ruin theory to credit risk. Rev. Quant. Finance Accounting 32, $373-403$.

Chiu, S. N. AND YIN, C. C. (2003). The time of ruin, the surplus prior to ruin and the deficit at ruin for the classical risk process perturbed by diffusion. Insurance Math. Econom. 33, 59-66.

Collin-Dufresne, P. and Goldstein, R. S. (2001). Do credit spreads reflect stationary leverage ratios? J. Finance 56, 1929-1957.

Dufresne, F. And Gerber, H. U. (1991). Risk theory for the compound Poisson process that is perturbed by diffusion. Insurance Math. Econom. 10, 51-59.

Dynkin, E. B. (1965). Markov Processes, Vols. I, II. Springer, Berlin.

FURRER, H. J. AND SCHMIDLI, H. (1994). Exponential inequalities for ruin probabilities of risk processes perturbed by diffusion. Insurance Math. Econom. 15, 23-36.

Gerber, H. U. (1970). An extension of the renewal equation and its application in the collective theory of risk. Skand. Aktuarietidskrift 1970, 205-210.

Gerber, H. U. ANd Shiu, E. S. W. (1998). On the time value of ruin. N. Amer. Actuarial J. 2, 48-78.

Gerber, H. U. and Shiu, E. S. W. (2005). The time value of ruin in a Sparre Andersen model. N. Amer. Actuarial J. 9, 49-84.

Hilberink, B. And Rogers, L. C. G. (2002). Optimal capital structure and endogenous default. Finance Stoch. 6, $237-263$.

HuANG, J.-Z. AND HuANG, M. (2003). How much of the corporate-treasury yield spread is due to credit risk? Working paper. Available at http://ssrn.com/abstract=307360.

HuANG, J.-Z. AND ZHOU, H. (2008). Specification analysis of structural credit risk models. Working paper. Available at http://ssrn.com/paper=1105640.

Kou, S. G. (2002). A jump-diffusion model for option pricing. Manag. Sci. 48, 1086-1101.

Kou, S. G. AND Wang, H. (2003). First passage times of a jump diffusion process. Adv. Appl. Prob. 35, 504-531.

Kou, S. G. And Wang, H. (2004). Option pricing under a double exponential jump diffusion model. Manag. Sci. 50, $1178-1192$.

Le Courtois, O. and QuitTard-Pinon, F. (2006). Risk-neutral and default probabilities with an endogenous bankruptcy jump-diffusion model. Asia-Pacific Financial Markets 13, 11-39.

Lindskog, F. And McNeIl, A. J. (2003). Common Poisson shock models: applications to insurance and credit risk modelling. ASTIN Bull. 33, 209-238.

LONGSTAFF, F. A. AND Schwartz, E. S. (1995). A simple approach to valuing risky and floating rate debt. J. Finance 50, 789-819.

Merton, R. C. (1974). On the pricing of corporate debt: the risk structure of interest rates. J. Finance 29, 449-470.

RAMEZANi, C. AND ZENG, Y. (2007). Maximum likelihood estimation of the double exponential jump-diffusion process. Ann. Finance 3, 487-507.

Revuz, D. AND Yor, M. (1991). Continuous Martingales and Brownian Motion. Springer, Berlin.

Tsai, C. C.-L. And Willmot, G. E. (2002). On the moments of the surplus process perturbed by diffusion. Insurance Math. Econom. 31, 327-350.

WANG, G. AND WU, R. (2000). Some distributions for classical risk process that is perturbed by diffusion. Insurance Math. Econom. 26, 15-24.

WANG, G. AND WU, R. (2008). The expected discounted penalty function for the perturbed compound Poisson risk process with constant interest. Insurance Math. Econom. 42, 59-64.

Yuen, K. C., Lu, L. AND Wu, R. (2009). The compound Poisson process perturbed by a diffusion with a threshold dividend strategy. Appl. Stoch. Models Business Industry 25, 73-93.

ZHou, C. (2001). The term structure of credit spreads with jump risk. J. Banking Finance 25, 2015-2040. 